\title{
Precision measurement of the mass of the $\tau$ lepton
}

M. Ablikim, ${ }^{1}$ M. N. Achasov, ${ }^{8, a}$ X. C. Ai, ${ }^{1}$ O. Albayrak, ${ }^{4}$ M. Albrecht, ${ }^{3}$ D. J. Ambrose ${ }^{41}$ F. F. An, ${ }^{1}$ Q. An ${ }^{42}$ J.Z. Bai, ${ }^{1}$ R. Baldini Ferroli, ${ }^{19 a}$ Y. Ban, ${ }^{28}$ J. V. Bennett, ${ }^{18}$ M. Bertani, ${ }^{19 a}$ J. M. Bian, ${ }^{40}$ E. Boger, ${ }^{21, b}$ O. Bondarenko, ${ }^{22}$ I. Boyko, ${ }^{21}$ S. Braun, ${ }^{37}$ R. A. Briere, ${ }^{4}$ H. Cai,${ }^{47}$ X. Cai, ${ }^{1}$ O. Cakir ${ }^{36 a}$ A. Calcaterra, ${ }^{19 a}$ G. F. Cao, ${ }^{1}$ S. A. Cetin, ${ }^{36 b}$ J. F. Chang, ${ }^{1}$ G. Chelkov, ${ }^{21, b}$ G. Chen, ${ }^{1}$ H. S. Chen, ${ }^{1}$ J. C. Chen, ${ }^{1}$ M. L. Chen, ${ }^{1}$ S. J. Chen, ${ }^{26}$ X. Chen, ${ }^{1}$ X. R. Chen, ${ }^{23}$ Y. B. Chen, ${ }^{1}$ H. P. Cheng, ${ }^{16}$ X. K. Chu, ${ }^{28}$ Y. P. Chu, ${ }^{1}$ D. Cronin-Hennessy, ${ }^{40}$ H. L. Dai, ${ }^{1}$ J. P. Dai, ${ }^{1}$ D. Dedovich,${ }^{21}$ Z. Y. Deng, ${ }^{1}$ A. Denig, ${ }^{20}$ I. Denysenko, ${ }^{21}$ M. Destefanis, ${ }^{45 a, 45 c}$ W. M. Ding, ${ }^{30}$ Y. Ding, ${ }^{24}$ C. Dong, ${ }^{27}$ J. Dong, ${ }^{1}$ L. Y. Dong, ${ }^{1}$ M. Y. Dong, ${ }^{1}$ S. X. Du, ${ }^{49}$ J. Z. Fan, ${ }^{35}$ J. Fang, ${ }^{1}$ S. S. Fang, ${ }^{1}$ Y. Fang, ${ }^{1}$ L. Fava, ${ }^{45 b, 45 c}$ C. Q. Feng, ${ }^{42}$ C. D. Fu, ${ }^{1}$ O. Fuks, ${ }^{21, b}$ Q. Gao, ${ }^{1}$ Y. Gao ${ }^{35}$ C. Geng, ${ }^{42}$ K. Goetzen, ${ }^{9}$ W. X. Gong, ${ }^{1}$ W. Gradl, ${ }^{20}$ M. Greco, ${ }^{45 a, 45 c}$ M. H. Gu, ${ }^{1}$ Y. T. Gu, ${ }^{11}$ Y. H. Guan, ${ }^{1}$ A. Q. Guo, ${ }^{27}$ L. B. Guo, ${ }^{25}$ T. Guo ${ }^{25}$ Y. P. Guo, ${ }^{20}$ Y. P. Guo, ${ }^{27}$ Y. L. Han,${ }^{1}$ F. A. Harris, ${ }^{39}$ K. L. He, ${ }^{1}$ M. He, ${ }^{1}$ Z. Y. He, ${ }^{27}$ T. Held, ${ }^{3}$ Y. K. Heng, ${ }^{1}$ Z. L. Hou, ${ }^{1}$ C. Hu, ${ }^{25}$ H. M. Hu, ${ }^{1}$ J. F. Hu ${ }^{37}$ T. Hu, ${ }^{1}$ G. M. Huang, ${ }^{5}$ G. S. Huang, ${ }^{42}$ H. P. Huang, ${ }^{47}$ J. S. Huang, ${ }^{14}$ L. Huang, ${ }^{1}$ X. T. Huang ${ }^{30}{ }^{3}$ Y. Huang,${ }^{26}$ T. Hussain, ${ }^{44}$ C. S. Ji, ${ }^{42}$ Q. Ji, ${ }^{1}$ Q. P. Ji, ${ }^{27}$ X. B. Ji, ${ }^{1}$ X. L. Ji, ${ }^{1}$ L. L. Jiang, ${ }^{1}$ L. W. Jiang, ${ }^{47}$ X. S. Jiang, ${ }^{1}$ J. B. Jiao, ${ }^{30}$ Z. Jiao, ${ }^{16}$ D. P. Jin, ${ }^{1}$ S. Jin, ${ }^{1}$ T. Johansson, ${ }^{46}$ N. Kalantar-Nayestanaki ${ }^{22}$ X. L. Kang, ${ }^{1}$ X. S. Kang, ${ }^{27}$ M. Kavatsyuk, ${ }^{22}$ B. Kloss, ${ }^{20}$ B. Kopf,${ }^{3}$ M. Kornicer, ${ }^{39}$ W. Kuehn, ${ }^{37}$ A. Kupsc, ${ }^{46}$ W. Lai, ${ }^{1}$ J. S. Lange, ${ }^{37}$ M. Lara, ${ }^{18}$ P. Larin, ${ }^{13}$ M. Leyhe, ${ }^{3}$ C. H. Li, ${ }^{1}$ Cheng Li, ${ }^{42}$ Cui Li,${ }^{42}$ D. Li, ${ }^{17}$ D. M. Li, ${ }^{49}{ }^{7}$. Li, ${ }^{1}$ G. Li, ${ }^{1}$ H. B. Li ${ }^{1}$ J. C. Li ${ }^{1}$

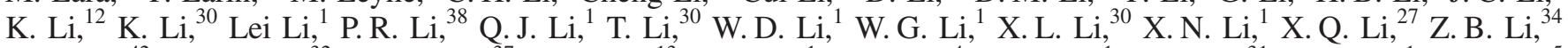
H. Liang, ${ }^{42}$ Y. F. Liang, ${ }^{32}$ Y. T. Liang, ${ }^{37}$ D. X. Lin, ${ }^{13}$ B. J. Liu, ${ }^{1}$ C. L. Liu, ${ }^{4}$ C. X. Liu, ${ }^{1}$ F. H. Liu, ${ }^{31}$ Fang Liu, ${ }^{1}$ Feng Liu ${ }^{5}$ H. B. Liu, ${ }^{11}$ H. H. Liu, ${ }^{15}$ H. M. Liu, ${ }^{1}$ J. Liu, ${ }^{1}$ J. P. Liu, ${ }^{47}$ K. Liu, ${ }^{35}$ K. Y. Liu, ${ }^{24}$ P. L. Liu, ${ }^{30}$ Q. Liu, ${ }^{38}$ S. B. Liu, ${ }^{42}$ X. Liu, ${ }^{23}$ Y. B. Liu, ${ }^{27}$ Z. A. Liu, ${ }^{1}$ Zhiqiang Liu, ${ }^{1}$ Zhiqing Liu, ${ }^{20}$ H. Loehner, ${ }^{22}$ X. C. Lou, ${ }^{1, c}$ G. R. Lu, ${ }^{14}$ H. J. Lu, ${ }^{16}$ H. L. Lu, ${ }^{1}$ J. G. Lu, ${ }^{1}$ X. R. Lu, ${ }^{38}$ Y. Lu, ${ }^{1}$ Y. P. Lu, ${ }^{1}$ C. L. Luo, ${ }^{25}$ M. X. Luo, ${ }^{48}$ T. Luo, ${ }^{39}$ X. L. Luo, ${ }^{1}$ M. Lv, ${ }^{1}$ F. C. Ma, ${ }^{24}$ H. L. Ma, ${ }^{1}$ Q. M. Ma, ${ }^{1}$ S. Ma, ${ }_{1}^{1}$ T. Ma, ${ }^{1}$ X. Y. Ma, ${ }^{1}$ F. E. Maas, ${ }^{13}$ M. Maggiora ${ }^{45 a, 45 c}$ Q. A. Malik, ${ }^{44}$ Y. J. Mao, ${ }^{28}$ Z. P. Mao, ${ }^{1}$ J. G. Messchendorp, ${ }^{22}$ J. Min, ${ }^{1}$ T. J. Min, ${ }^{1}$ R. E. Mitchell,${ }^{18}$ X. H. Mo ${ }^{1}$ Y. J. Mo,${ }^{5}$ H. Moeini, ${ }^{22}$ C. Morales Morales, ${ }^{13}$ K. Moriya, ${ }^{18}$

N. Yu. Muchnoi, ${ }^{8, a}$ H. Muramatsu, ${ }^{40}$ Y. Nefedov ${ }^{21}$ I. B. Nikolaev, ${ }^{8, a}$ Z. Ning, ${ }^{1}$ S. Nisar, ${ }^{7}$ X. Y. Niu, ${ }^{1}$ S. L. Olsen, ${ }^{29}$ Q. Ouyang, ${ }^{1}$ S. Pacetti ${ }_{19 b}$ M. Pelizaeus, ${ }^{3}$ H. P. Peng, ${ }^{42}$ K. Peters, ${ }^{9}$ J. L. Ping, ${ }_{1}{ }^{25}$ R. G. Ping, ${ }^{1}$ R. Poling,${ }^{40}$ N. Q.. ${ }^{47}$ M. Qi ${ }^{26}$ S. Qian, ${ }^{1}$ C. F. Qiao, ${ }^{38}$ L. Q. Qin, ${ }^{30}$ X. S. Qin, ${ }^{1}$ Y. Qin, ${ }^{28}$ Z. H. Qin, ${ }^{1}$ J. F. Qiu, ${ }^{1}$ K. H. Rashid,${ }^{44}$ C. F. Redmer, ${ }^{20}$ M. Ripka, ${ }^{20}$ G. Rong, ${ }^{1}$ X. D. Ruan, ${ }^{11}$ A. Sarantsev ${ }^{21, d}$ K. Schoenning, ${ }^{46}$ S. Schumann, ${ }^{20}$ W. Shan, ${ }^{28}$ M. Shao,${ }^{42}$ C. P. Shen, ${ }^{2}$ X. Y. Shen, ${ }^{1}$ H. Y. Sheng, ${ }^{1}$ M. R. Shepherd, ${ }^{18}$ W. M. Song, ${ }^{1}$ X. Y. Song, ${ }^{1}$ S. Spataro, ${ }^{45},{ }^{4}{ }^{45}$ B. Spruck, ${ }^{37}$ G. X. Sun, ${ }^{1}$ J. F. Sun, ${ }^{14}$ S. S. Sun ${ }^{1}$ Y. J. Sun, ${ }^{42}$ Y. Z. Sun, ${ }^{1}$ Z. J. Sun, ${ }^{1}$ Z. T. Sun, ${ }^{42}$ C. J. Tang,${ }^{32}$ X. Tang, ${ }^{1}$ I. Tapan, ${ }^{36 c}$ E. H. Thorndike,${ }^{41}$ D. Toth, ${ }^{40}$ M. Ullrich, ${ }^{37}$ I. Uman, ${ }^{36 b}$ G. S. Varner, ${ }^{39}$ B. Wang, ${ }^{27}$ D. Wang, ${ }^{28}$ D. Y. Wang, ${ }^{28}$ K. Wang, ${ }^{1}$ L. L. Wang, ${ }^{1}$ L. S. Wang, ${ }_{1}^{1}$ M. Wang, ${ }^{30}$ P. Wang, ${ }^{1}$ P. L. Wang, ${ }^{1}$ Q. J. Wang, ${ }^{1}$ S. G. Wang, ${ }^{28}$ W. Wang, ${ }^{1}$ X. F. Wang, ${ }^{35}{ }^{2}$ Y. D. Wang, ${ }^{19 a}$ Y. F. Wang, ${ }^{1}$ Y. Q. Wang, ${ }^{20}$ Z. Wang, ${ }^{1}$ Z. G. Wang, ${ }^{1}$ Z. H. Wang, ${ }^{42}$ Z. Y. Wang, ${ }^{1}$ D. H. Wei, ${ }^{10}$ J. B. Wei ${ }^{28}$ P. Weidenkaff, ${ }^{20}$ S. P. Wen, ${ }^{1}$ M. Werner, ${ }^{37}$ U. Wiedner, ${ }^{3}$ M. Wolke, ${ }^{46}$ L. H. Wu, ${ }^{1}$ N. Wu, ${ }^{1}$ Z. Wu, ${ }^{1}$ L. G. Xia, ${ }^{35}$ Y. Xia,${ }^{17}$ D. Xiao, ${ }^{1}$ Z. J. Xiao, ${ }^{25}$ Y. G. Xie, ${ }^{1}$ Q. L. Xiu, ${ }^{1}$ G. F. Xu, ${ }^{1}$ L. Xu, ${ }^{1}$ Q. J. Xu, ${ }^{12}$ Q. N. Xu, ${ }^{38}$ X. P. Xu ${ }^{33}$ Z. Xue, ${ }^{1}$ L. Yan, ${ }^{42}$ W. B. Yan, ${ }^{42}$ W. C. Yan, ${ }^{42}$ Y. H. Yan, ${ }^{17}$ H. X. Yang, ${ }^{1}$ L. Yang, ${ }^{47}$ Y. Yang, ${ }^{5}$ Y. X. Yang, ${ }^{10}$ H. Ye, ${ }^{1}$ M. Ye, ${ }^{1}$ M. H. Ye, ${ }^{6}$ B.X. Yu, ${ }^{1}$ C. X. Yu, ${ }^{27}$ H.W. Yu, ${ }^{28}$ J. S. Yu, ${ }^{23}$ S. P. Yu, ${ }_{1}{ }^{30}$ C. Z. Yuan, ${ }^{1}$ W. L. Yuan, ${ }^{26}$ Y. Yuan, ${ }^{1}$ A. A. Zafar, ${ }^{44}$ A. Zallo, ${ }^{19 a}$ S. L. Zang, ${ }^{26}$ Y. Zeng, ${ }^{17}$ B. X. Zhang, ${ }^{1}$ B. Y. Zhang, ${ }^{1}$

C. Zhang, ${ }^{26}$ C. B. Zhang, ${ }^{17}$ C. C. Zhang, ${ }^{1}$ D. H. Zhang, ${ }^{1}$ H. H. Zhang, ${ }^{34}$ H. Y. Zhang, ${ }^{1}$ J. J. Zhang, ${ }^{1}$ J. Q. Zhang, ${ }^{1}$

J. W. Zhang, ${ }^{1}$ J. Y. Zhang, ${ }^{1}$ J. Z. Zhang, ${ }^{1}$ S. H. Zhang, ${ }^{1}$ X. J. Zhang, ${ }^{1}$ X. Y. Zhang, ${ }^{30}$ Y. Zhang, ${ }^{1}$ Y. H. Zhang, ${ }^{1}$ Z. H. Zhang, ${ }^{5}$ Z. P. Zhang, ${ }^{42}$ Z. Y. Zhang, ${ }^{47}$ G. Zhao, ${ }^{1}$ J. W. Zhao, ${ }^{1}$ Lei Zhao, ${ }^{42}$ Ling Zhao, ${ }^{1}$ M. G. Zhao, ${ }^{27}$ Q. Zhao, ${ }^{1}$ Q. W. Zhao,

S. J. Zhao, ${ }^{49}$ T. C. Zhao, ${ }^{1}$ X. H. Zhao, ${ }^{26}$ Y. B. Zhao, ${ }^{1}$ Z. G. Zhao, ${ }^{42}$ A. Zhemchugov, ${ }^{21, b}$ B. Zheng, ${ }^{43}$ J. P. Zheng, ${ }^{1}$

Y. H. Zheng, ${ }^{38}$ B. Zhong, ${ }^{25}$ L. Zhou, ${ }^{1}$ Li Zhou, ${ }^{27}$ X. Zhou, ${ }^{47}$ X. K. Zhou, ${ }^{38}$ X. R. Zhou, ${ }^{42}$ X. Y. Zhou, ${ }^{1}$ K. Zhu, ${ }^{1}$ K. J. Zhu, ${ }^{1}$ X. L. Zhu, ${ }^{35}$ Y. C. Zhu, ${ }^{42}$ Y. S. Zhu, ${ }^{1}$ Z. A. Zhu, ${ }^{1}$ J. Zhuang, ${ }^{1}$ B. S. Zou, ${ }^{1}$ and J. H. Zou ${ }^{1}$

\section{(BESIII Collaboration)}

\author{
${ }^{1}$ Institute of High Energy Physics, Beijing 100049, People's Republic of China \\ ${ }^{2}$ Beihang University, Beijing 100191, People's Republic of China \\ ${ }^{3}$ Bochum Ruhr-University, D-44780 Bochum, Germany \\ ${ }^{4}$ Carnegie Mellon University, Pittsburgh, Pennsylvania 15213, USA \\ ${ }^{5}$ Central China Normal University, Wuhan 430079, People's Republic of China \\ ${ }^{6}$ China Center of Advanced Science and Technology, Beijing 100190, People's Republic of China \\ ${ }^{7}$ COMSATS Institute of Information Technology, Lahore, Defence Road, \\ Off Raiwind Road, 54000 Lahore, Pakistan \\ ${ }^{8}$ G.I. Budker Institute of Nuclear Physics SB RAS (BINP), Novosibirsk 630090, Russia \\ ${ }^{9}$ GSI Helmholtzcentre for Heavy Ion Research GmbH, D-64291 Darmstadt, Germany \\ ${ }^{10}$ Guangxi Normal University, Guilin 541004, People's Republic of China \\ ${ }^{11}$ GuangXi University, Nanning 530004, People's Republic of China
}




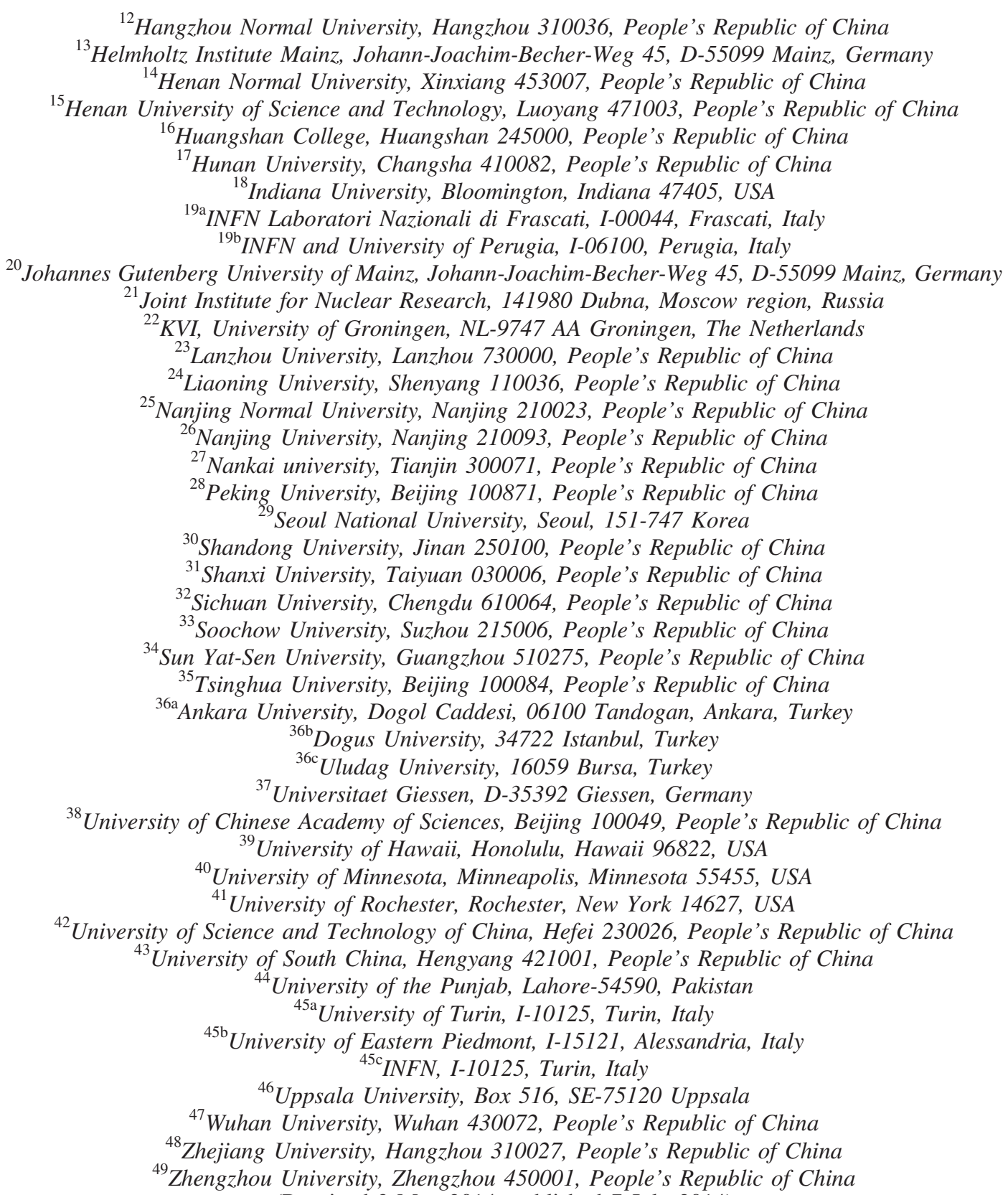

(Received 3 May 2014; published 7 July 2014)

An energy scan near the $\tau$ pair production threshold has been performed using the BESIII detector. About $24 \mathrm{pb}^{-1}$ of data, distributed over four scan points, were collected. This analysis is based on $\tau$ pair decays to $e e, e \mu, e h, \mu \mu, \mu h, h h, e \rho, \mu \rho$ and $\pi \rho$ final states, where $h$ denotes a charged $\pi$ or $K$. The mass of the $\tau$ lepton is measured from a maximum likelihood fit to the $\tau$ pair production cross-section data to be $m_{\tau}=\left(1776.91 \pm 0.12_{-0.13}^{+0.10}\right) \mathrm{MeV} / c^{2}$, which is currently the most precise value in a single measurement.

DOI: 10.1103/PhysRevD.90.012001

PACS numbers: 14.60.Fg, 13.35.Dx

\footnotetext{
${ }^{a}$ Also at the Novosibirsk State University, Novosibirsk 630090, Russia.

${ }^{b}$ Also at the Moscow Institute of Physics and Technology, Moscow 141700, Russia.

${ }^{c}$ Also at University of Texas at Dallas, Richardson, Texas 75083, USA.

${ }^{\mathrm{d}}$ Also at the PNPI, Gatchina 188300, Russia.
}

\section{INTRODUCTION}

The $\tau$ lepton mass, $m_{\tau}$, is one of the fundamental parameters of the Standard Model (SM). The relationship between the $\tau$ lifetime $\left(\tau_{\tau}\right)$, mass, its electronic branching 
fraction $(B(\tau \rightarrow e \nu \bar{\nu}))$ and weak coupling constant $g_{\tau}$ is predicted by theory,

$$
\frac{B(\tau \rightarrow e \nu \bar{\nu})}{\tau_{\tau}}=\frac{g_{\tau}^{2} m_{\tau}^{5}}{192 \pi^{3}}
$$

up to small radiative and electroweak corrections [1]. It appeared to be badly violated before the first precise $m_{\tau}$ measurement of BES became available in 1992 [2]; this measurement was later updated with more $\tau$ decay channels [3] and confirmed by subsequent measurements from BELLE [4], KEDR [5], and BABAR [6]. The experimental determination of $\tau_{\tau}, B(\tau \rightarrow e \nu \bar{\nu})$ and $m_{\tau}$ to the highest possible precision is essential for a high precision test of the SM. Currently, the mass precision for $e$ and $\mu$ has reached $\Delta m / m$ of $10^{-8}$, while for $\tau$ it is $10^{-4}$ [7].

A precision $m_{\tau}$ measurement is also required to check lepton universality. Lepton universality, a basic ingredient in the minimal standard model, requires that the chargedcurrent gauge coupling strengths $g_{e}, g_{\mu}, g_{\tau}$ should be identical: $g_{e}=g_{\mu}=g_{\tau}$. Comparing the electronic branching fractions of $\tau$ and $\mu$, lepton universality can be tested as

$$
\left(\frac{g_{\tau}}{g_{\mu}}\right)^{2}=\frac{\tau_{\mu}}{\tau_{\tau}}\left(\frac{m_{\mu}}{m_{\tau}}\right)^{5} \frac{B(\tau \rightarrow e \nu \bar{\nu})}{B(\mu \rightarrow e \nu \bar{\nu})}\left(1+F_{W}\right)\left(1+F_{\gamma}\right),
$$

where $F_{W}$ and $F_{\gamma}$ are the weak and electromagnetic radiative corrections [1]. Note $\left(g_{\tau} / g_{\mu}\right)^{2}$ depends on $m_{\tau}$ to the fifth power.

Furthermore, the precision of $m_{\tau}$ will also restrict the ultimate sensitivity of $m_{\nu_{\tau}}$. The most sensitive bounds on the mass of the $\nu_{\tau}$ can be derived from the analysis of the invariant-mass spectrum of semi-hadronic $\tau$ decays, e.g. the present best limit of $m_{\nu_{\tau}}<18.2 \mathrm{MeV} / c^{2}$ (95\% confidence level) was based on the kinematics of 2939 (52) events of $\tau^{-} \rightarrow 2 \pi^{-} \pi^{+} \nu_{\tau}\left(\tau^{-} \rightarrow 3 \pi^{-} 2 \pi^{+}\left(\pi^{-}\right) \nu_{\tau}\right)$ [8]. This method depends on a determination of the kinematic end point of the mass spectrum; thus high precision on $m_{\tau}$ is needed.

So far, the pseudomass technique and the threshold scan method have been used to determine $m_{\tau}$. The former, which was used by ARGUS [9], OPAL [10], BELLE [4] and $B A B A R$ [6], relies on the reconstruction of the invariantmass and energy of the hadronic system in the hadronic $\tau$ decay, while the latter, which was used in DELCO [11], BES [2,3], and KEDR [5], is a study of the threshold behavior of the $\tau$ pair production cross section in $e^{+} e^{-}$ collisions and it is the method used in this paper. Extremely important in this approach is to determine the beam energy and the beam energy spread precisely. Here the beam energy measurement system (BEMS) [12] for BEPCII is used and will be described below.

Before the experiment began, a study was carried out using Monte Carlo (MC) simulation and sampling to optimize the number and choice of scan points in order to provide the highest precision on $m_{\tau}$ for a specified period of data taking time or equivalently for a given integrated luminosity [13].

The $\tau$ scan experiment was done in December 2011. The $J / \psi$ and $\psi^{\prime}$ resonances were each scanned at seven energy points, and data were collected at four scan points near $\tau$ pair production threshold with center of mass (c.m.) energies of $3542.4,3553.8,3561.1$ and $3600.2 \mathrm{MeV}$. The first $\tau$ scan point is below the mass of $\tau$ pair [7], while the other three are above.

\section{BESIII DETECTOR}

The BESIII detector is designed to study hadron spectroscopy and $\tau$-charm physics [14]. The cylindrical BESIII is composed of (1) A helium-gas-based main drift chamber (MDC) with 43 layers providing an average single-hit resolution of $135 \mu \mathrm{m}$ and a charged-particle momentum resolution in a $1 \mathrm{~T}$ magnetic field of $0.5 \%$ at $1.0 \mathrm{GeV} / c$, (2) a time-of-flight (TOF) system constructed of 5-cm-thick plastic scintillators, with 176 counters of $2.4 \mathrm{~m}$ length in two layers in the barrel and 96 fan-shaped counters in the end caps. The barrel (end cap) time resolution of $80 \mathrm{ps}$ (110 ps) provides $2 \sigma K / \pi$ separation for momenta up to $1.0 \mathrm{GeV} / c$. (3) A CsI(Tl) Electro-Magnetic Calorimeter (EMC) consisting of 6240 crystals in a cylindrical barrel structure and two end caps. The energy resolution at $1.0 \mathrm{GeV} / c$ is $2.5 \%(5 \%)$ in the barrel (end caps), while the position resolution is $6 \mathrm{~mm}(9 \mathrm{~mm})$ in the barrel (end caps). (4) A Resistive plate chamber (RPC)-based muon chamber (MUC) consisting of $1000 \mathrm{~m}^{2}$ of RPCs in nine barrel and eight end cap layers and providing $2 \mathrm{~cm}$ position resolution.

\section{BEAM ENERGY MEASUREMENT SYSTEM}

\section{A. Introduction}

The BEMS is located at the north crossing point of the BEPCII storage ring. The layout schematic of BEMS is shown in Fig. 1. This design allows us to measure the energies of both the electron and positron beams with one laser and one high-purity germanium (HPGe) detector [12].

In the Compton scattering process, the maximal energy of the scattered photon $E_{\gamma}$ is related to the electron energy $E_{e}$ by the kinematics of Compton scattering [15,16],

$$
E_{e}=\frac{E_{\gamma}}{2}\left[1+\sqrt{1+\frac{m_{e}^{2}}{\mathcal{E}_{\gamma} E_{\gamma}}}\right],
$$

where $\mathcal{E}_{\gamma}$ is the energy of the initial photon, i.e. the energy of the laser beam in the BEMS. The scattered photon energy can be measured with high accuracy by the HPGe detector, whose energy scale is calibrated with photons 


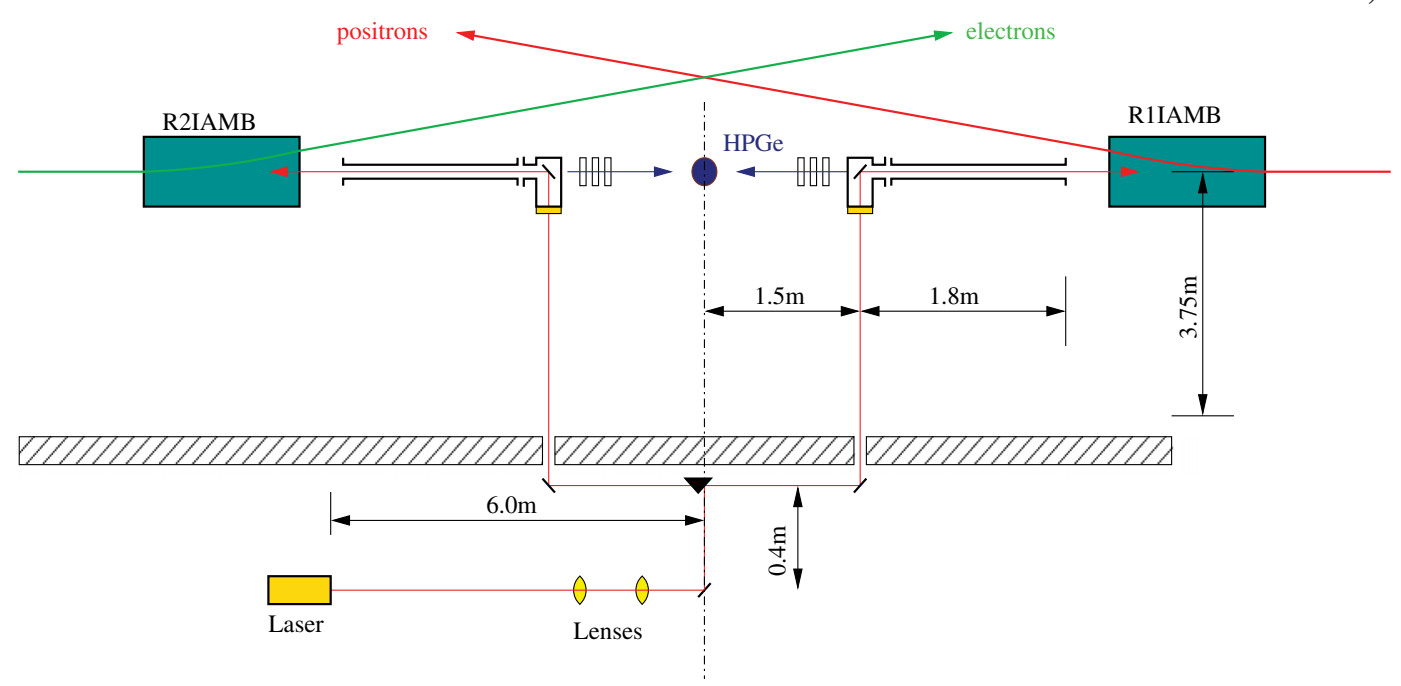

FIG. 1 (color online). Simplified schematic of the beam energy measurement system. The positron and electron beams are indicated. R1IAMB and R2IAMB are accelerator magnets, and the HPGe detector is represented by the dot at the center. The half-meter shielding wall of the beam tunnel is shown crosshatched. The laser and optics system is located outside the tunnel of the storage ring, where the optics system is composed of two lenses, mirrors and a prism denoted by the inverted solid triangle.

from radioactive sources and the readout linearity is checked with a precision pulser. The maximum energy can be determined from the fitting to the edge of the scattered photon energy spectrum. At the same time the energy spread of back-scattered photons due to the energy distribution of the collider beam is obtained from the fitting procedure [12]. Finally, the electron energy can be calculated by Eq. (3). Since the energy of the laser beam and the electron mass $\left(m_{e}\right)$ are determined with the accuracy at the level of $10^{-8}, E_{e}$ can be determined, utilizing Eq. (3), as accurately as $E_{\gamma}$, an accuracy is at the level of $10^{-5}$. The systematic error of the electron and positron beam energy determination in our experiment was tested through previous measurement of the $\psi^{\prime}$ mass and was estimated as $2 \times 10^{-5}$ [12]; the relative uncertainty of the beam energy spread was about $6 \%$ [12].

\section{B. Determination of scan-point energy}

The BEMS alternates between measuring electron and positron beam energies, and writes out energy calibration (EC) data files. Each EC file has its own time stamp that can be used to associate BEMS measurements with corresponding scan data. In the $\tau$-scan region, all EC runs within the start and end times of a scan point are used for determining the scan-point energy.

In the case of fast energy scans in the $J / \psi$ and $\psi^{\prime}$ resonance regions, the ratio of hadronic and Bhabha events is used to determine scan-point boundaries. Once all electron and positron EC files that belong to a particular scan point are grouped, we determine the c.m. energy of the crossing beams at the given scan point by using the errorweighted average of electron and positron beam energies,
$\bar{E}_{e^{-}}$and $\bar{E}_{e^{+}}$, respectively. The c.m. energy of a scan point is calculated using

$$
E_{\mathrm{c} . \mathrm{m} .}=2 \sqrt{\bar{E}_{e^{-}} \cdot \bar{E}_{e^{+}}} \cdot \cos \left(\theta_{e^{+} e^{-}} / 2\right),
$$

where $\theta_{e^{+} e^{-}}=0.022 \mathrm{rad}$ is the crossing angle between the beams. Table I gives measured luminosities $(\mathcal{L})$ at each scan point, in which the c.m. energy is obtained from Eq. (4); the method to determine these luminosities will be introduced in Sec. VI A.

TABLE I. Measured integrated luminosities at each scan point. The errors are statistical only.

\begin{tabular}{lcc}
\hline \hline Scan & $E_{\text {c.m. }}(\mathrm{MeV})$ & $\mathcal{L}\left(\mathrm{nb}^{-1}\right)$ \\
\hline$J / \psi$ & 3088.7 & $78.5 \pm 1.9$ \\
& 3095.3 & $219.3 \pm 3.1$ \\
& 3096.7 & $243.1 \pm 3.3$ \\
& 3097.6 & $206.5 \pm 3.1$ \\
& 3098.3 & $223.5 \pm 3.2$ \\
& 3098.8 & $216.9 \pm 3.1$ \\
& 3103.9 & $317.3 \pm 3.8$ \\
$\tau$ & 3542.4 & $4252.1 \pm 18.9$ \\
& 3553.8 & $5566.7 \pm 22.8$ \\
& 3561.1 & $3889.2 \pm 17.9$ \\
& 3600.2 & $9553.0 \pm 33.8$ \\
$\psi^{\prime}$ & 3675.9 & $787.0 \pm 7.2$ \\
& 3683.7 & $823.1 \pm 7.4$ \\
& 3685.1 & $832.4 \pm 7.5$ \\
& 3686.3 & $1184.3 \pm 9.1$ \\
& 3687.6 & $1660.7 \pm 11.0$ \\
& 3688.8 & $767.7 \pm 7.2$ \\
& 3693.5 & $1470.8 \pm 10.3$ \\
\hline \hline
\end{tabular}



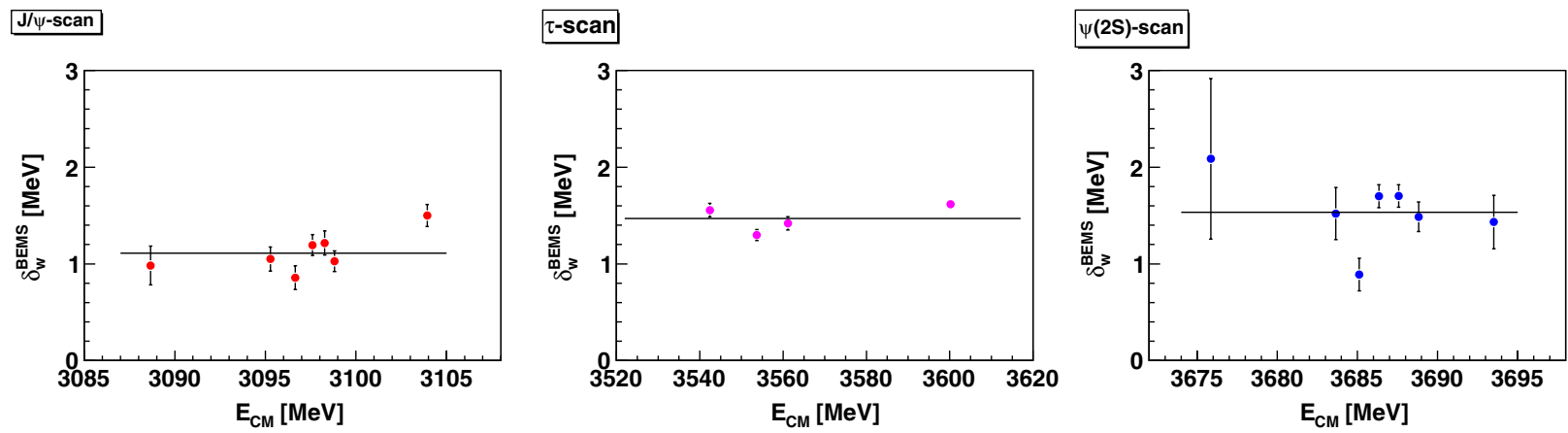

FIG. 2 (color online). Energy spreads from the $J / \psi$ (left), $\tau$ (middle) and $\psi^{\prime}$ scans (right). Horizontal lines represent mean values, listed in Table II.

\section{Determination of beam energy spread}

Besides measuring the energy of the electron or positron beams, the BEMS also measures the energy spread independently of the energy measurements by the accelerator. Using the same grouping of the EC data, we obtain weighted averages of the electron, $\delta_{e^{-}}$, and the positron, $\delta_{e^{+}}$, energy spreads. Corresponding errors, $\Delta\left(\delta_{e^{-}}\right)$and $\Delta\left(\delta_{e^{+}}\right)$, represent one standard deviation of weighted averages. Taking into account that the beam energy has a Gaussian distribution around its mean with the width given by the energy spread, the total energy spread of a scan point, $\delta_{w}^{\mathrm{BEMS}}$, is calculated from the average electron and positron spreads using

$$
\delta_{w}^{\mathrm{BEMS}}=\sqrt{\delta_{e^{-}}^{2}+\delta_{e^{+}}^{2}}
$$

It is assumed that the $e^{-}$and $e^{+} \mathrm{EC}$ measurements are independent and that the total beam energy spread results from the sum of two uncorrelated Gaussian distributions. The crossing angle between the beams has little effect on the energy spread and is ignored. Consequently, the error on the total spread is obtained using error propagation:

$$
\Delta\left(\delta_{w}^{\mathrm{BEMS}}\right)=\sqrt{\delta_{e_{-}}^{2} \cdot \Delta^{2}\left(\delta_{e_{-}}\right)+\delta_{e_{+}}^{2} \cdot \Delta^{2}\left(\delta_{e_{+}}\right)} / \delta_{w}^{\mathrm{BEMS}} .
$$

Figure 2 shows the corresponding energy spreads from the $J / \psi$ (left), $\tau$ (middle) and $\psi^{\prime}$ (right) scan regions. The spreads show little dependence on energy within a given scan region, and increase gradually as the c.m. energy increases from the $J / \psi$ to the $\psi^{\prime}$ region. Because of the large fluctuations, the energy spread in each scan region is

TABLE II. Energy spreads $(\mathrm{MeV})$ from the $J / \psi, \tau$ and $\psi^{\prime}$ scan regions calculated using Eqs. (5) and (6).

\begin{tabular}{lcc}
\hline \hline Scan & $\delta_{w}^{\text {BEMS }}$ & $\Delta\left(\delta_{w}^{\text {BEMS }}\right)$ \\
\hline$J / \psi$ & 1.112 & 0.070 \\
$\tau$ & 1.469 & 0.064 \\
$\psi^{\prime}$ & 1.534 & 0.109 \\
\hline \hline
\end{tabular}

estimated by calculating the mean energy spread, taking the error as one standard deviation of the mean. The mean values are summarized in Table II, and shown as horizontal lines on the plots in Fig. 2.

\section{THE DATA SAMPLE AND MC SIMULATION}

The $J / \psi$ and $\psi^{\prime}$ scan data samples listed in Table I are used to determine the line shape of each resonance, and the parameters obtained are used to validate the BEMS measurements. All of the data collected near the $\tau$ pair production threshold are used to do the $\tau$ mass measurement.

The luminosity at each scan point is determined using two-gamma events $\left[e^{+}+e^{-} \rightarrow \gamma \gamma(\gamma)\right]$. Bhabha events are used to do a cross-check. The Babayaga 3.5 generator [17] is used as our primary generator.

To devise selection criteria for hadronic events in resonance scans, we analyzed $\approx 10^{6}$ events from the $J / \psi$ and $\psi^{\prime}$ data and $\approx 50 \times 10^{6}$ events from the continuum data produced at center-of-mass energies $3096 \mathrm{MeV}, 3686 \mathrm{MeV}$ and $3650 \mathrm{MeV}$, respectively. Approximately $5 \times 10^{6}$ events from corresponding $J / \psi$ and $\psi^{\prime}$ inclusive MC samples are also used to optimize the selection criteria.

The GEANT4-based [18] simulation software, BESIII Object Oriented Simulation [19], contains the detector geometry and material description, the detector response and signal digitization models, as well as the detector running conditions and performance. The production of the $J / \psi$ and $\psi^{\prime}$ resonance is simulated by the Monte Carlo event generator KKMC [20]; the known decay modes are generated by EVTGEN [21] with branching ratios set at Particle Data Group (PDG) [7] world average values and by LUNDCHARM [22] for the remaining unknown decays.

KKMC [20] is also used to simulate the production of $\tau$ pairs, and EVTGEN [21] is used to generate all $\tau$ decay modes with branching ratios set at PDG [7] world average values. The MC sample including all of the possible decay channels is used as the $\tau$ pair inclusive MC sample, while the sample including only a specific decay channel is used as a $\tau$ exclusive MC sample. 


\section{EVENT SELECTION CRITERIA}

Four data samples, including two-gamma events, Bhabha events, hadronic events and $\tau$ pair candidate events, are used in this analysis. Selection criteria to select these samples with high efficiency while removing background are listed below.

\section{A. Good photon selection criteria}

A neutral cluster is considered to be a good photon candidate if the deposited energy is larger than $25 \mathrm{MeV}$ in the barrel EMC $(|\cos \theta|<0.8)$ or $50 \mathrm{MeV}$ in the end cap $\operatorname{EMC}(0.86<|\cos \theta|<0.92)$, where $\theta$ is the polar angle of the shower.

\section{B. Good charged track election criteria}

Good charged tracks are required to satisfy $V_{r}=$ $\sqrt{V_{x}^{2}+V_{y}^{2}}<1 \mathrm{~cm},\left|V_{z}\right|<10 \mathrm{~cm}$. Here $V_{x}, V_{y}$ and $V_{z}$ are the $x, y$ and $z$ coordinates of the point of closest approach to the interaction point (IP), respectively. The track is also required to lie within the region $|\cos \theta|<0.93$.

\section{Two-gamma events}

The number of good photons is required to be larger than one and less than eleven; the energy of the highest energy photon must be larger than $0.85 \times E_{\mathrm{c} . \mathrm{m} .} / 2$ and less than $1.1 \times E_{\text {c.m. }} / 2$; the energy of the second highest energy photon must be larger than $0.57 \times E_{\text {c.m. }} / 2$; and the difference of the azimuthal angles of the two highest energy photons in the c.m. must satisfy $176^{\circ}<\Delta \phi<183^{\circ}$. It is also required that there are no charged tracks in the events.

\section{Bhabha events}

The charged tracks must satisfy $V_{r}<2 \mathrm{~cm},\left|V_{z}\right|<10 \mathrm{~cm}$, $|\cos \theta|<0.80$ and have momenta $p<2500 \mathrm{MeV} / c$. A good photon must have deposited energy in the EMC less than $1.1 \times E_{\text {c.m. }} / 2$ and have $0<t<750 \mathrm{~ns}$, where $t$ is the time information from the EMC, to suppress electronic noise and energy deposits unrelated to the event. For the whole event, the following selection criteria are used: the visible energy of the event must be larger than $0.22 \times E_{\text {c.m. }}$, the number of charged tracks is required to be two or three, the momentum of the highest momentum charged track must be larger than $0.65 \times E_{\mathrm{c} . \mathrm{m}} / 2$, the ratio $E / c p$ of one of the two highest momentum tracks must be larger than 0.6, where $E$ is the energy deposited in the calorimeter and $p$ is the track momentum determined by the MDC, and the difference of the azimuthal angles of the two high momentum tracks in the c.m. system must satisfy $175^{\circ}<\Delta \phi<185^{\circ}$.

\section{E. Hadronic events}

Aside from standard selection requirements for good charged tracks, the average vertex position along the beam line is required to satisfy $\left|\bar{V}_{Z}\right|=\left|\frac{\sum_{i}^{N_{c h}} V_{z}^{i}}{N_{c h}}\right|<4 \mathrm{~cm}$, and the number of charged tracks $\left(N_{c h}\right)$ must be larger than two.

\section{F. $\tau$ pair candidate events}

In order to reduce the statistical error in the $\tau$ lepton mass, this analysis incorporates 13 two-prong $\tau$ pair final states, which are $e e, e \mu, e \pi, e K, \mu \mu, \mu \pi, \mu K, \pi K, \pi \pi, K K, e \rho, \mu \rho$ and $\pi \rho$, with accompanying neutrinos implied. For the first ten decay channels, there is no photon; for $X \rho(X=e, \mu$ or $\pi)$, the $\rho$ candidate is reconstructed with $\pi^{ \pm} \pi^{0}$, so there are two photons in the final state. No photons are allowed except in the $\rho$ case where only two are allowed. The number of good charged tracks and also the number of total charged tracks are required to be two for all channels. The following event selection criteria are applied to both data and MC samples.

\section{Additional requirements on good photons}

Apart from those basic requirements, good photons must have the angle between the cluster and the nearest charged particle larger than 20 degrees. Also we require $0<t<750 \mathrm{~ns}$.

\section{PID for each charged track}

For each charged track, the measured $p, E, E / c p$, the time-of-flight value, the depth of the track in the MUC $(D)$ and the total number of hits in the MUC $\left(N_{h}\right)$ are used together to identify the particle type; the particle identification (PID) criteria are listed in Table III. In this table,

TABLE III. PID for charged particles. For the first scan point, the values of $p_{\min }\left(p_{\max }\right)$ are $0.2(0.92), 0.2(0.9), 0.84(0.93)$, and $0.76 \mathrm{GeV} / c(0.88 \mathrm{GeV} / c)$ for $e, \mu, \pi$, and $K$, respectively.

\begin{tabular}{lcccc}
\hline \hline PID & $p(\mathrm{MeV} / c)$ & EMC & TOF & MUC \\
\hline$e$ & $p_{\text {min }}<p<p_{\text {max }}$ & $0.8<E / c p<1.05$ & $|\Delta \mathrm{TOF}(e)|<0.2 \mathrm{~ns}$ & \\
& & $0 \mathrm{~ns}<\mathrm{TOF}<4.5 \mathrm{~ns}$ & \\
$\mu$ & $p_{\text {min }}<p<p_{\max }$ & $E / c p<0.7$ & $|\Delta \mathrm{TOF}(\mu)|<0.2 \mathrm{~ns}$ & $(D>(80 \times p-50) \mathrm{cm}$ or $D>40 \mathrm{~cm})$ \\
& & $0.1<E<0.3$ & $|\Delta \mathrm{TOF}(\pi)|<0.2 \mathrm{~ns}$ & and $N_{h}>1$ \\
$\pi$ & $p_{\text {min }}<p<p_{\max }$ & $E / c p<0.6$ & $0 \mathrm{~ns}<\mathrm{TOF}<4.5 \mathrm{~ns}$ & not $\mu$ \\
& & & $|\Delta \mathrm{TOF}(K)|<0.2 \mathrm{~ns}$ & not $\mu$ \\
$K$ & $p_{\text {min }}<p<p_{\max }$ & $E / c p<0.6$ & $0 \mathrm{~ns}<\mathrm{TOF}<4.5 \mathrm{~ns}$ & \\
\hline \hline
\end{tabular}


$\Delta \operatorname{TOF}(e)$ is the difference between the calculated timeof-flight of the track when it is assigned as an electron and the time-of-flight measured by TOF; $\Delta \mathrm{TOF}(\mu)$, $\Delta \operatorname{TOF}(\pi)$ and $\Delta \operatorname{TOF}(K)$ are similar quantities. $p_{\min }$ and $p_{\max }$ are the minimum and maximum momentum of charged tracks in any $\tau$ decay at a given c.m. energy, which are all determined from the signal MC simulation and are different in different scan energy points as $p$ of these daughter particles are related with the initial momentum of $\tau^{ \pm}$. For $\pi^{ \pm}$from $\rho^{ \pm}$, the $p$ requirement is removed for the PID.

\section{Other additional requirements}

For the $X \rho$ channels, the invariant mass of the two photons $[M(\gamma \gamma)]$ is required to be in the $\pi^{0}$ mass window which is $[112.8,146.4] \mathrm{MeV} / c^{2}$. Then these two photons are used together with a $\pi$ candidate to reconstruct a $\rho$ candidate, and the invariant-mass of the $\rho$ candidate is required to be in the mass window i.e. $[376.5,1195.5] \mathrm{MeV} / c^{2}$. Also, the magnitude of the momentum of the $\rho$ candidate must be more than the minimum expected momentum $\left(p_{\min }^{\rho}\right)$ and less than the maximum $\left(p_{\max }^{\rho}\right)$, where $p_{\min }^{\rho}$ and $p_{\max }^{\rho}$ are also determined from the $p$ distribution of $\rho$ candidates in the signal MC samples.

The $\tau$ pair candidate $e e$ event sample contains background from two-photon $e^{+} e^{-} \rightarrow e^{+}\left(e^{-} e^{+}\right) e^{-}$events in which the leading $e^{+}$and $e^{-}$in the final state are undetected. These QED background events are characterized by small net observed transverse momentum and large missing energy. It follows that the variable PTEM, defined as

$$
\mathrm{PTEM}=\frac{P_{T}}{E_{\text {miss }}^{\max }}=\frac{\left(c \vec{P}_{1}+c \vec{P}_{2}\right)_{T}}{W-\left|c \vec{P}_{1}\right|-\left|c \vec{P}_{2}\right|},
$$

which is the ratio of the net observed transverse momentum to the maximum possible value of the missing energy, is localized to small values for QED background events. The first point in the $\tau$ mass scan experiment is located below the $\tau$ pair production threshold (about 11.2 MeV below the mass of the $\tau$ pair, where the $\tau$ mass from the PDG is used), so all events passing the criteria for selecting $\tau$ pair candidates at this point are background, and can be used to study the event selection criteria and the background level at the same time. The correlation between PTEM and the acoplanarity angle $\theta_{\text {acop }}$ is studied for the background data set and the signal MC sample. The acoplanarity angle $\theta_{\text {acop }}$ is defined as the angle between the planes spanned by the beam direction and the momentum vectors of the two final state charged tracks; i.e., it is the angle between the transverse momentum vectors of the two final state charged tracks. Figures 3(a) and 3(b) are the distributions of PTEM versus $\theta_{\text {acop }}$ for $e e$ candidate events from the first scan energy point data set, and $e e$ events from the $\tau$ pair MC
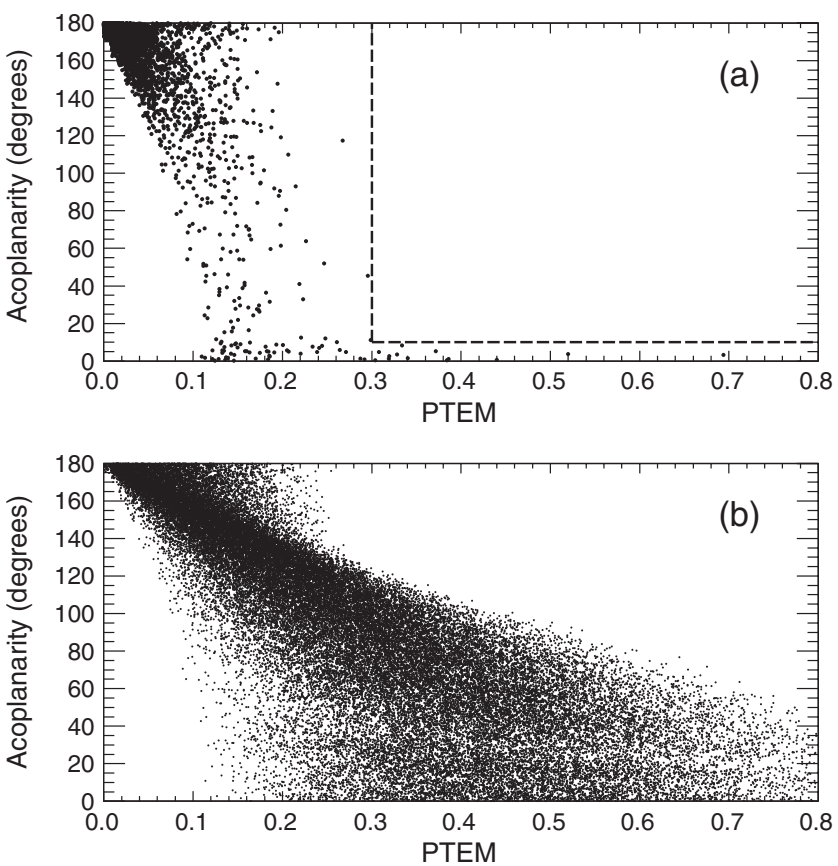

FIG. 3. (a) The scatter plot of PTEM versus acoplanarity for the $\tau$ pair candidate $e e$ event from the first scan energy point, which is below $\tau$ pair production threshold. The region above and to the right of the dashed line are the acceptance region. (b) The same scatter plot for $e e$ events obtained from the $\tau$ pair MC simulation corresponding to the second scan point, which is above $\tau$ pair production threshold, after applying the same selection criteria as used for data.

sample corresponding to the second scan point, respectively.

From the comparison of these two plots, we retain only those $e e$ events having PTEM $>0.3$ and $\theta_{\text {acop }}>10^{\circ}$. By comparing the scatter plots of PTEM versus $\theta_{\text {acop }}$ from the first scan-point data set and that from the second scan- point signal MC simulation sample, we obtain similar requirements for the other $\tau$ pair decay channels, which are listed in Table IV.

TABLE IV. Selection requirements on acoplanarity angle and PTEM for different final states.

\begin{tabular}{lcr}
\hline \hline final state & $\theta_{\text {acop }}$ & PTEM \\
\hline$e e$ & $>10^{\circ}$ & $>0.3$ \\
$e \mu$ & $<160^{\circ}$ & $>0.1$ \\
$e \pi$ & $<170^{\circ}$ & $>0.1$ \\
$e K$ & $<170^{\circ}$ & \\
$\mu \mu$ & $<140^{\circ}$ & \\
$\mu h$ & $<140^{\circ}$ & \\
$h h$ & $<160^{\circ}$ & \\
$e \rho$ & $<170^{\circ}$ & \\
$\mu \rho$ & $<150^{\circ}$ & \\
$\pi \rho$ & & \\
\hline \hline
\end{tabular}




\section{DATA ANALYSIS}

\section{A. Luminosity at each scan point}

For all scan points, the luminosity $\mathcal{L}$ is determined from $\mathcal{L}=N_{\text {data }} / \epsilon_{\gamma \gamma} \sigma_{\gamma \gamma}$, where $N_{\text {data }}$ is the number of selected two-gamma events in data and $\epsilon_{\gamma \gamma}$ and $\sigma_{\gamma \gamma}$ are the efficiency and the cross section determined by the Babayaga 3.5 MC, respectively. The measured luminosity $(\mathcal{L})$ at each scan point is listed in Table I, from which the integrated luminosities for the $J / \psi, \tau$ and $\psi^{\prime}$ scan are calculated as 1505,23261 , and $7526 \mathrm{nb}^{-1}$, respectively. The analysis using Bhabha events is done as a cross-check of the twogamma luminosity and gives consistent luminosity results within $2 \%$. The Bhabha luminosities will also be used in the systematic error analyses.

\section{B. $J / \psi$ and $\psi^{\prime}$ hadronic cross-section line shapes}

The number of hadronic events $N^{h}$ is fitted to the number of expected hadronic events,

$$
N^{\exp }=\sigma_{\text {had }} \cdot \mathcal{L},
$$

where $\sigma_{\text {had }}$ is the cross section of $e^{+} e^{-} \rightarrow$ hadrons, which depends on $E_{\text {c.m. }}$, and the energy spread, $\delta_{w}$,

$$
\sigma_{\text {had }}\left(E_{\text {c.m. }}, \delta_{w}\right)=\sigma_{\text {bg }} \cdot\left(\frac{M}{E_{\text {c.m. }}}\right)^{2}+\epsilon_{\text {had }} \cdot \sigma_{\text {res }}\left(E_{\text {c.m. }}, M, \delta_{w}\right) .
$$

Here, $M$ is the resonance mass, and the resonance cross section, $\sigma_{\text {res }}$, is obtained from the hadronic cross section, $\sigma_{0}$, described in Ref. [23], taking into account radiative corrections. The hadronic cross section is convoluted with a Gaussian with a width equal to the beam energy spread:

$$
\sigma_{\text {rez }}=\int_{-\infty}^{+\infty} \frac{e^{-\frac{1}{2}\left(\frac{E_{c, m},-E_{\text {c.m. }} \cdot}{\delta_{w_{w}}}\right)^{2}}}{\sqrt{2 \pi} \delta_{w}} \sigma_{0}\left(E_{\text {c.m. }}^{\prime}, M\right) d E_{\text {c.m. }}^{\prime} .
$$

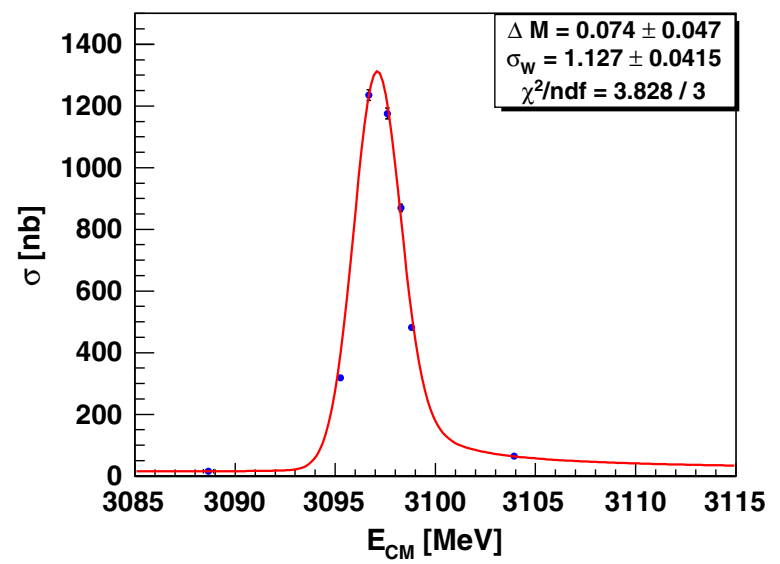

The background cross section, $\sigma_{\mathrm{bg}}$, reconstruction efficiency, $\epsilon_{\text {had }}, M$, and $\delta_{w}$ are free parameters, obtained from minimizing

$$
\chi^{2}=\sum_{i=1}^{N} \frac{\left(N_{i}^{h}-\sigma_{\text {had }}^{i} \mathcal{L}_{i}\right)^{2}}{N_{i}^{h}\left(1+N_{i}^{h}\left(\Delta \mathcal{L}_{i} / \mathcal{L}_{i}\right)^{2}\right)},
$$

where $\Delta \mathcal{L}_{i} / \mathcal{L}_{i}, N_{i}^{h}, \sigma_{\text {had }}^{i}$ and $\mathcal{L}_{i}$ are the relative luminosity error, the number of hadron events, the cross section of $e^{+} e^{-} \rightarrow$ hadrons, and the luminosity at scan point $i$, respectively. Figure 4 shows the number of hadronic events from the $J / \psi$ (left) and $\psi^{\prime}$ (right) regions, fitted to the hadronic cross-section line shapes given by Eq. (10). The mass difference with respect to nominal resonance mass, $\Delta M=M^{\mathrm{fit}}-M^{\mathrm{PDG}}$, and the energy spread from the $J / \psi$ and $\psi^{\prime}$ fits are given in Table V, where the first error is statistical and the second is systematic. The values for $\delta_{w}$ in Table V agree well with those in Table II obtained from the BEMS.

The systematic errors are determined by applying different selection criteria on the number of hadronic events, and using the Bhabha luminosity instead of the two-gamma luminosity. In addition, systematic errors from fitting resonance line-shapes when background is allowed to interfere (Ref. [24]) are taken into account. The systematic error associated with determining scan-point energies from the EC data, estimated by comparing calibration lines and pulsing lines in the BEMS system, is negligible compared to statistical errors on c.m. energies.

We extrapolate the results from the fits of the $J / \psi$ and $\psi^{\prime}$ line shapes to the $\tau$-mass region in order to obtain the energy correction to the $\tau$-mass. The systematic error associated with the energy scale is estimated by extrapolating under two assumptions: the first one is that the correction has a linear dependence on the energy and the second one assumes a constant shift. The linear fit between data points from Table $\mathrm{V}$ gives the correction to the $\tau$-mass of $\Delta m_{\tau}=(0.054 \pm 0.030) \mathrm{MeV} / c^{2}$; the constant shift

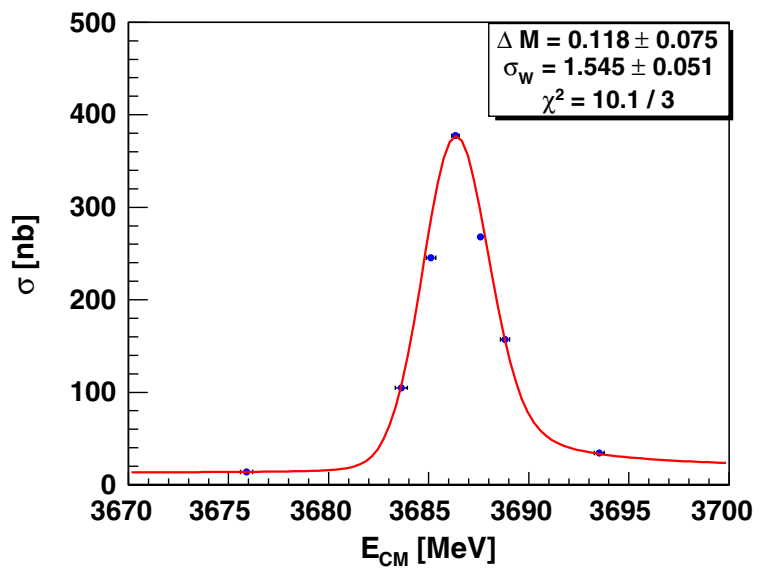

FIG. 4 (color online). Fits of the $J / \psi$ (left) and $\psi^{\prime}$ (right) hadronic cross sections. 
TABLE V. Fit parameters from the $J / \psi$ and $\psi^{\prime}$ fits, where the first error is statistical and the second is systematic. $\Delta M$ is the difference between fitted $\psi$ mass and the normal value from PDG. All units are in $\mathrm{MeV} / c^{2}$.

\begin{tabular}{lcc}
\hline \hline Scan & $\Delta M$ & $\delta_{w}$ \\
\hline$J / \psi$ & $0.074 \pm 0.047 \pm 0.043$ & $1.127 \pm 0.042 \pm 0.050$ \\
$\psi^{\prime}$ & $0.118 \pm 0.076 \pm 0.021$ & $1.545 \pm 0.051 \pm 0.069$ \\
\hline \hline
\end{tabular}

gives the correction of $\Delta m_{\tau}=(0.043 \pm 0.020) \mathrm{MeV} / c^{2}$, where both errors are statistical. The difference between these two methods, $0.011 \mathrm{MeV} / c^{2}$, is taken as the systematic uncertainty related to the energy determination. The difference of $0.005 \mathrm{MeV}$ from taking into account background interference when fitting the $J / \psi$ and $\psi^{\prime}$ line shapes is taken as an additional systematic uncertainty. Overall, the systematic error in the energy determination is taken to be $0.012 \mathrm{MeV}$. The difference

$$
\Delta m_{\tau}=0.054 \pm 0.030 \text { (stat) } \pm 0.012 \text { (sys) } \mathrm{MeV} / c^{2} \text {, }
$$

will be taken into consideration in the measured $\tau$-mass value.

\section{C. $\tau$ Mass Measurement}

\section{Comparison of the data and MC samples}

The comparison between the number of final $\tau$ pair candidate events from data and from the $\tau$ pair inclusive MC samples are listed in Table VI ordered by final state and scan point, where the indexes in the first row, from 1 to 4 , represent the index of the scan points. The $\tau$ pair inclusive MC sample has been normalized to the data according to the luminosity at each point, and the numbers of normalized MC events have been multiplied by the ratio of the overall efficiencies for identifying $\tau$ pair events for data and MC simulation, which is fitted from the data set in the following section.

The comparison of some distributions between data and the $\tau$ pair inclusive MC samples are shown in Fig. 5. These comparisons and those in Table VI indicate that data and MC samples agree well with each other.

The selected $\tau$ pair candidate events are used for the measurement of the mass of the $\tau$ lepton and the corresponding $\tau$ pair inclusive MC samples are used to obtain the selection efficiency for different decay channels.

\section{Maximum likelihood fit to the data}

The mass of the $\tau$ lepton is obtained from a maximum likelihood fit to the c.m. energy dependence of the $\tau$ pair production cross section. The likelihood function is constructed from Poisson distributions, one at each of the four scan points, and takes the form [3]

$$
L\left(m_{\tau}, \mathcal{R}_{\mathrm{data} / \mathrm{MC}}, \sigma_{B}\right)=\prod_{i=1}^{4} \frac{\mu_{i}^{N_{i}} e^{-\mu_{i}}}{N_{i} !},
$$

where $N_{i}$ is the number of observed $\tau$ pair events at scan point $i ; \mu_{i}$ is the expected number of events and calculated by

$$
\mu_{i}=\left[\mathcal{R}_{\mathrm{data} / \mathrm{MC}} \times \epsilon_{i} \times \sigma\left(E_{\mathrm{c} . \mathrm{m} .}^{i}, m_{\tau}\right)+\sigma_{B}\right] \times \mathcal{L}_{i} .
$$

In Eq. (14), $m_{\tau}$ is the mass of the $\tau$ lepton, and $\mathcal{R}_{\text {data/MC }}$ is the ratio of the overall efficiency for identifying $\tau$ pair events for data and for MC simulation, allowing for the

TABLE VI. A comparison of the numbers of events by final state to those from the $\tau$ pair inclusive MC sample. The MC sample has

\begin{tabular}{|c|c|c|c|c|c|c|c|c|c|c|}
\hline \multirow[b]{2}{*}{ Final state } & \multicolumn{2}{|c|}{1} & \multicolumn{2}{|c|}{2} & \multicolumn{2}{|c|}{3} & \multicolumn{2}{|c|}{4} & \multicolumn{2}{|c|}{ Total } \\
\hline & Data & MC & Data & $\mathrm{MC}$ & Data & MC & Data & MC & Data & MC \\
\hline$e e$ & 0 & 0 & 4 & 3.7 & 13 & 12.2 & 84 & 76.1 & 101 & 92.0 \\
\hline$e \mu$ & 0 & 0 & 8 & 9.1 & 35 & 31.4 & 168 & 192.6 & 211 & 233.1 \\
\hline$e \pi$ & 0 & 0 & 8 & 8.6 & 33 & 29.7 & 202 & 184.4 & 243 & 222.6 \\
\hline$e K$ & 0 & 0 & 0 & 0.5 & 2 & 1.8 & 16 & 16.9 & 18 & 19.3 \\
\hline$\mu \mu$ & 0 & 0 & 2 & 2.9 & 8 & 9.2 & 49 & 56.3 & 59 & 68.4 \\
\hline$\mu \pi$ & 0 & 0 & 4 & 3.9 & 11 & 14.1 & 89 & 86.7 & 104 & 104.7 \\
\hline$\mu K$ & 0 & 0 & 0 & 0.2 & 3 & 0.8 & 7 & 9.0 & 10 & 10.1 \\
\hline$\pi \pi$ & 0 & 0 & 1 & 2.0 & 5 & 7.7 & 57 & 54.0 & 63 & 63.8 \\
\hline$\pi K$ & 0 & 0 & 1 & 0.3 & 0 & 0.8 & 10 & 8.2 & 11 & 9.3 \\
\hline$K K$ & 0 & 0 & 0 & 0.0 & 1 & 0.1 & 1 & 0.3 & 2 & 0.4 \\
\hline$e \rho$ & 0 & 0 & 3 & 6.1 & 19 & 20.6 & 142 & 132.0 & 164 & 158.7 \\
\hline$\mu \rho$ & 0 & 0 & 8 & 3.3 & 8 & 11.8 & 52 & 63.3 & 68 & 78.5 \\
\hline$\pi \rho$ & 0 & 0 & 5 & 3.4 & 15 & 10.8 & 97 & 96.0 & 117 & 110.2 \\
\hline Total & 0 & 0 & 44 & 44.2 & 153 & 151.2 & 974 & 975.7 & 1171 & 1171.0 \\
\hline
\end{tabular}
been normalized to the data according to the luminosity at each point, and the numbers of normalized MC events have been multiplied by the ratio of the overall efficiencies for identifying $\tau$ pair events for data and MC simulation. 

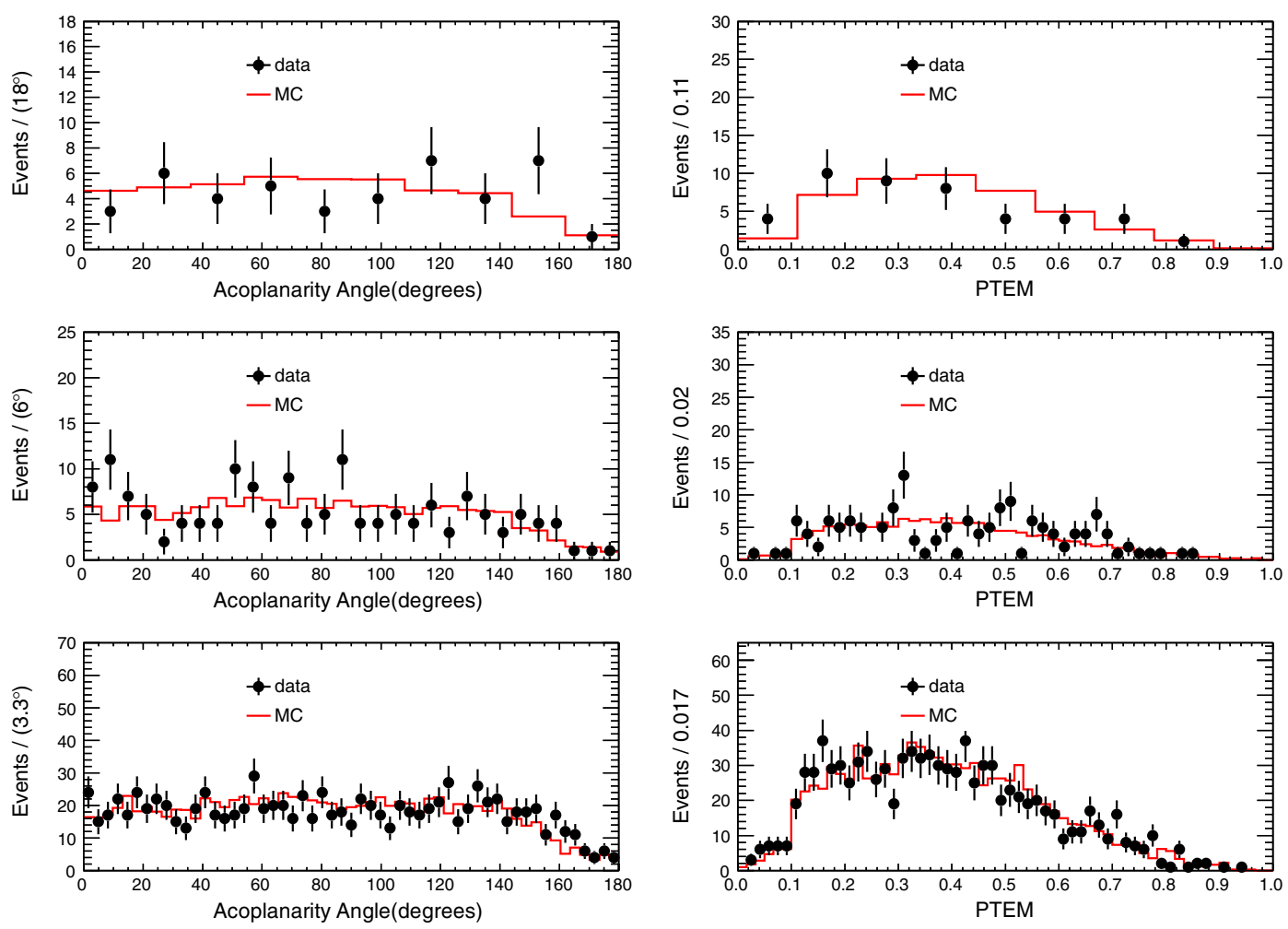

FIG. 5 (color online). (left) The distribution in acoplanarity angle between two charged tracks and (right) the distribution in PTEM. Dots with error bars are data and the histogram is $\tau$ pair inclusive MC. The upper two plots are from the second scan point, the middle two are from the third scan point, and the lower two are from the fourth scan point.

difference of the efficiencies between the data and the corresponding MC sample. $\epsilon_{i}$ is the efficiency at scan point $i$, which is given by $\epsilon_{i}=\sum_{i} B r_{j} \epsilon_{i j}$, where $B r_{j}$ is the branching fraction for the $j$ th final state and $\epsilon_{i j}$ is the detection efficiency for the $j$ th final state at the $i$ th scan point. The efficiencies $\epsilon_{i}$, determined directly from the $\tau$ pair inclusive MC sample by applying the same $\tau$ pair selection criteria, are $0.065,0.065,0.069,0.073$ at the four scan points, respectively. $\sigma_{B}$ is an effective background cross section, and it is assumed constant over the limited range of c.m. energy, $E_{\text {c.m. }}^{i}$, covered by the scan. $\mathcal{L}_{i}$ is the integrated luminosity at scan point $i$, and $\sigma\left(E_{\text {c.m. }}^{i}, m_{\tau}\right)$ is the corresponding cross section for $\tau$ pair production which has the form [3]

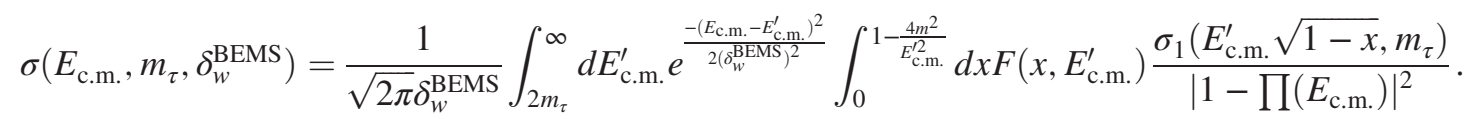

Here, $\delta_{w}^{\text {BEMS }}$ is the c.m. energy spread, determined from the BEMS, $F\left(x, E_{\mathrm{c} . \mathrm{m}}\right)$ is the initial state radiation factor [25], $\prod\left(E_{\text {c.m. }}\right)$ is the vacuum polarization factor $[24,26,27]$, and $\sigma_{1}\left(E_{\mathrm{c} . \mathrm{m}}, m_{\tau}\right)$ is the high accuracy, improved cross section from Voloshin [28]. In carrying out the maximum likelihood (ML) fit, $m_{\tau}, \mathcal{R}_{\text {data/MC }}$ and $\sigma_{B}$ are allowed to vary, subject to the requirement $\sigma_{B} \geq 0$.

To test the procedure, the likelihood fit is performed on the selected $\tau$ pair inclusive MC data sample. The input $m_{\tau}$ is $1776.90 \mathrm{MeV} / \mathrm{c}^{2}$, while the fitted value of $m_{\tau}$ is found to be $m_{\tau}=(1776.90 \pm 0.12) \mathrm{MeV} / c^{2}$; the good agreement between the input and output values indicates that the fitting procedure is reliable.
The same ML fit is performed on the selected $\tau$ pair candidate events. The fit yields

$$
\begin{aligned}
m_{\tau} & =1776.91 \pm 0.12 \mathrm{MeV} / c^{2}, \\
\mathcal{R}_{\text {data } / \mathrm{MC}} & =1.05 \pm 0.04, \\
\sigma_{B} & =0^{+0.12} \mathrm{pb} .
\end{aligned}
$$

The fitted $\sigma_{B}$ is zero, which indicates the selected $\tau$ pair candidate data set is very pure.

The quality of the fit is shown explicitly in Fig. 6 (left plot). The curve corresponds to the cross section given by Eq. (15) with $m_{\tau}=1776.91 \mathrm{MeV} / c^{2}$; the measured cross section at scan point $i$ is given by 

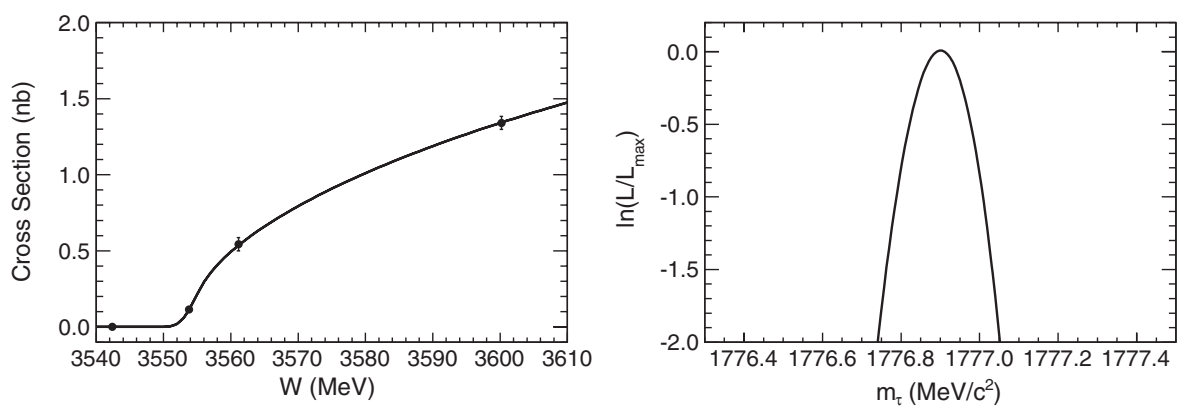

FIG. 6. (left) The c.m. energy dependence of the $\tau$ pair cross section resulting from the likelihood fit (curve), compared to the data (Poisson errors), and (right) the dependence of the logarithm of the likelihood function on $m_{\tau}$, with the efficiency and background parameters fixed at their most likely values.

$$
\sigma_{i}=\frac{N_{i}}{\mathcal{R}_{\text {data } / \mathrm{MC}} \epsilon_{i} \mathcal{L}_{i}}
$$

The measured cross sections at different scan points are consistent with the theoretical values. In Fig. 6 (right plot), the dependence of $\ln L$ on $m_{\tau}$ is almost symmetric as a consequence of the large data sample obtained.

\section{Systematic error estimation}

Theoretical accuracy.-The systematic error associated with the theoretical $\tau$ pair production cross section is estimated by comparing the difference of the fitted $m_{\tau}$ between two cases; in one case, the old $\tau$ pair production cross- section formulas are used, in the other, the improved version formulas are used. The uncertainty due to this effect is at the level of $10^{-3} \mathrm{MeV} / c^{2}$. More details can be found in Ref. [29].

Energy scale.-The $m_{\tau}$ shift, $\Delta \tau_{M}=(0.054 \pm 0.030$ (stat $) \pm$ 0.012 (syst)) $\mathrm{MeV} / c^{2}$ [Eq. (12)] is taken as a systematic error. Combining statistical and systematic errors, two boundaries can be established: $\Delta m_{\tau}^{\text {low }}=0.054-0.032=0.022 \mathrm{MeV} / c^{2}$ and $\Delta m_{\tau}^{\text {high }}=0.054+0.032=0.086 \mathrm{MeV} / c^{2}$. We take the higher value to form a negative systematic error and the lower value the positive systematic error. The systematic errors on the $m_{\tau}$ from this source are $\Delta m_{\tau}^{-}=0.086 \mathrm{MeV} / c^{2}$ and $\Delta m_{\tau}^{+}=0.022 \mathrm{MeV} / c^{2}$.

Energy spread.-From Table II, $\delta_{w}^{\mathrm{BEMS}}$ at the $\tau$ scan energy points is determined from the BEMS to be $(1.469 \pm$ $0.064) \mathrm{MeV}$. If we assume quadratic dependence of $\delta_{w}$ on energy, we can also extrapolate the $J / \psi$ and $\psi^{\prime}$ energy spreads to the $\tau$ region, which yields $\delta_{w}=(1.471 \pm$ $0.040) \mathrm{MeV}$. The difference of energy spreads obtained from these two methods is taken as a systematic uncertainty. The largest contribution to the energy spread uncertainty comes from interference effects. Including interference, the difference between the extrapolated value and the BEMS measurement is $0.056 \mathrm{MeV}$, and the overall systematic error is taken as $0.057 \mathrm{MeV}$. The final energy spread at the $\tau$ scan energy points is $(1.469 \pm 0.064 \pm$ $0.057) \mathrm{MeV}$. The uncertainty of $m_{\tau}$ from this item is estimated by refitting the data when the energy spread is set at its $\pm 1 \sigma$ values, and the shifted value of the fitted $m_{\tau}$, $\pm 0.016 \mathrm{MeV} / c^{2}$, is taken as the systematic error. Table VII lists the fitted results with different energy spread values.

Luminosity.-Both the Bhabha and the two-gamma luminosities are used in fitting the $\tau$ mass, and the difference of fitted $\tau$ masses is taken as the systematic error due to uncertainty in the luminosity determination. The difference is $0.001 \mathrm{MeV} / c^{2}$.

The $\tau$ mass shift [Eq. (12)] is $0.054 \mathrm{MeV} / c^{2}$ when determined with two-gamma luminosities. If Bhabha luminosities are used instead, the mass shift is $0.059 \mathrm{MeV} / c^{2}$, and the difference, $0.005 \mathrm{MeV} / c^{2}$, is also taken as a systematical error due to the luminosity. The total systematical uncertainty from luminosity determination is $0.006 \mathrm{MeV} / c^{2}$.

Number of good photons. - It is required that there are no extra good photons in our final states. Bhabha events are selected as a control sample to study the efficiency difference between data and $\mathrm{MC}$ of this requirement. The efficiency for data is $(79.17 \pm 0.06) \%$, and the efficiency for the MC simulation is $(79.01 \pm 0.14) \%$, where the errors are statistical. Correcting the number of observed events from data for the efficiency difference, we refit the $\tau$ mass, and the change of $\tau$ mass is $0.002 \mathrm{MeV} / c^{2}$, which is taken as the systematic uncertainty for this requirement.

TABLE VII. The $\tau$ mass determined from fits with different energy spreads.

\begin{tabular}{lc}
\hline \hline$\delta_{w}^{\text {BEMS }}(\mathrm{MeV})$ & $\tau$ mass $\left(\mathrm{MeV} / c^{2}\right)$ \\
\hline 1.383 & $1776.891_{-0.117}^{+0.111}$ \\
1.469 & $1776.906_{-0.1120}^{+0.116}$ \\
1.553 & $1776.919_{-0.126}^{+0.119}$ \\
\hline \hline
\end{tabular}


PTEM and acoplanarity angle requirements.-The nominal selection criteria on PTEM and acoplanarity angle, which are described in Sec. V F 3, are determined based on the first scan-point data. The $\tau$ mass is refitted using an alternative selection, where the requirements on PTEM and acoplanarity angle have been optimized based on MC simulation, and the change of the fitted $\tau$ mass from the nominal value, $0.05 \mathrm{MeV} / c^{2}$, is taken as the systematic error.

Mis-ID efficiency.-To determine the systematic error from misidentification between channels, two fits are done. In the first (nominal) fit, we use the particle ID efficiencies and misidentification (mis-ID) rates as obtained from $\tau$ pair inclusive MC samples. For the second fit, we extract PID efficiencies and mis-ID rates from selected data control samples of radiative Bhabha events, $J / \psi \rightarrow \rho \pi$, and cosmic ray events, correct the selection efficiencies of the different $\tau$ pair final states and propagate these changes to the event selection efficiencies $\epsilon_{i}$. We then refit our data with these modified efficiencies. The difference between the fitted $\tau$ mass from these two fits, $0.048 \mathrm{MeV} / c^{2}$, is taken as the systematic error due to misidentification between different channels.

Background shape.--In this analysis, the background cross section $\sigma_{B}$ is assumed to be constant for different $\tau$ scan points. The background cross sections have also been estimated at the last three scan points by applying their selection criteria on the first scan-point data, where the $\tau$ pair production is zero. After fixing $\sigma_{B}$ to these values, the fitted $\tau$ mass becomes

$$
m_{\tau}=(1776.87 \pm 0.12) \mathrm{MeV} / c^{2} .
$$

The fitted $\tau$ mass changed by $0.04 \mathrm{MeV} / c^{2}$ compared to the nominal result.

Fitted efficiency parameter.-The systematic uncertainties associated with the fitted efficiency parameter are obtained by setting $\mathcal{R}_{\text {data } / M C}$ at its $\pm 1 \sigma$ value and maximizing the

TABLE VIII. Summary of the $\tau$ mass systematic errors.

\begin{tabular}{lc}
\hline \hline Source & $\Delta m_{\tau}\left(\mathrm{MeV} / c^{2}\right)$ \\
\hline Theoretical accuracy & 0.010 \\
Energy scale & ${ }_{-0.022}^{+0.026}$ \\
Energy spread & 0.016 \\
Luminosity & 0.006 \\
Cut on number of good photons & 0.002 \\
Cuts on PTEM and acoplanarity angle & 0.05 \\
mis-ID efficiency & 0.048 \\
Background shape & 0.04 \\
Fitted efficiency parameter & +0.038 \\
Total & -0.034 \\
\hline \hline
\end{tabular}

likelihood with respect to $m_{\tau}$ with $\sigma_{B}=0$. This method yields changes in the fitted $\tau$ mass of $\Delta m_{\tau}={ }_{-0.034}^{+0.038} \mathrm{MeV}$, which is taken as the systematic uncertainty.

Total systematic error.-The systematic error sources and their contributions are summarized in Table VIII. We assume that all systematical uncertainties are independent and add them in quadrature to obtain the total systematical uncertainty for $\tau$ mass measurement, which is ${ }_{-0.13}^{+0.10} \mathrm{MeV} / c^{2}$.

\section{RESULTS}

By a maximum likelihood fit to the $\tau$ pair cross-section data near threshold, the mass of the $\tau$ lepton has been measured as

$$
m_{\tau}=\left(1776.91 \pm 0.12_{-0.13}^{+0.10}\right) \mathrm{MeV} / c^{2} .
$$

Figure 7 shows the comparison of measured $\tau$ mass in this paper with values from the PDG [7]; our result is consistent with all of them, but with the smallest uncertainty.

Using our $\tau$ mass value, together with the values of $B(\tau \rightarrow e \nu \bar{\nu})$ and $\tau_{\tau}$ from the PDG [7], we can calculate $g_{\tau}$ through Eq. (1),

$$
g_{\tau}=(1.1650 \pm 0.0034) \times 10^{-5} \mathrm{GeV}^{-2},
$$

which can be used to test the SM.

Similarly, inserting our $\tau$ mass value into Eq. (2), together with the values of $\tau_{\mu}, \tau_{\tau}, m_{\mu}, m_{\tau}, B(\tau \rightarrow e \nu \bar{\nu})$ and $B(\mu \rightarrow e \nu \bar{\nu})$ from the PDG [7] and using the values of $F_{W}(-0.0003)$ and $F_{\gamma}(0.0001)$ calculated from Ref. [1], the ratio of squared coupling constants is determined to be

$$
\left(\frac{g_{\tau}}{g_{\mu}}\right)^{2}=1.0016 \pm 0.0042,
$$

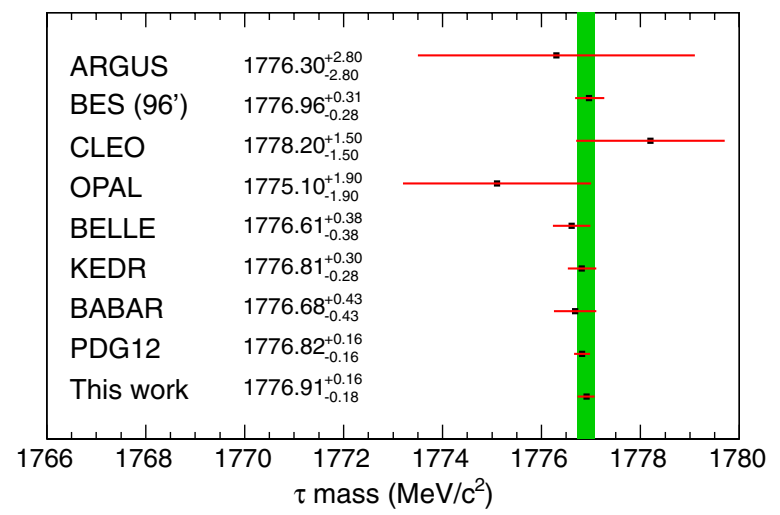

FIG. 7 (color online). Comparison of measured $\tau$ mass from this paper with those from the PDG. The green band corresponds to the $1 \sigma$ limit of the measurement of this paper. 
so that this test of lepton universality is satisfied at the 0.4 standard deviation level. The level of precision is compatible with previous determinations, which used the PDG average for $m_{\tau}$ [30].

\section{ACKNOWLEDGMENTS}

The BESIII Collaboration thanks the staff of BEPCII and the computing center for their strong support. This work is supported in part by the Ministry of Science and Technology of China under Contract No. 2009CB825200; Joint Funds of the National Natural Science Foundation of China under Contracts No. 11079008, No. 11179007, No. U1332201; National Natural Science Foundation of China (NSFC) under Contracts No. 11375206, No. 10625524, No. 10821063, No. 10825524,
No. 10835001, No. 10935007, No. 11125525, No. 11235011, No. 11179020; the Chinese Academy of Sciences (CAS) Large-Scale Scientific Facility Program; CAS under Contracts No. KJCX2-YW-N29, No. KJCX2YW-N45; 100 Talents Program of CAS; German Research Foundation DFG under Contract No. Collaborative Research Center CRC-1044; Istituto Nazionale di Fisica Nucleare, Italy; Ministry of Development of Turkey under Contract No. DPT2006K-120470; U.S. Department of Energy under Contracts No. DE-FG02-04ER41291, No. DE-FG02-05ER41374, No. DE-FG02-94ER40823, No. DESC0010118; U.S. National Science Foundation; University of Groningen (RuG) and the Helmholtzzentrum fuer Schwerionenforschung $\mathrm{GmbH}$ (GSI), Darmstadt; WCU Program of National Research Foundation of Korea under Contract No. R32-2008-000-10155-0.
[1] W. J. Marciano and A. Sirlin, Phys. Rev. Lett. 61, 1815 (1988).

[2] J. Z. Bai et al. (BES Collaboration), Phys. Rev. Lett. 69, 3021 (1992).

[3] J. Z. Bai et al. (BES Collaboration), Phys. Rev. D 53, 20 (1996).

[4] K. Belous et al. (Belle Collaboration), Phys. Rev. Lett. 99, 011801 (2007).

[5] V. V. Anashin et al. (KEDR Collaboration), JETP Lett. 85, 347 (2007).

[6] B. Aubert et al. (BABAR Collaboration), Phys. Rev. D 80, 092005 (2009)

[7] J. Beringer et al. (Particle Data Group), Phys. Rev. D 86, 010001 (2012) and 2013 partial update for the 2014 edition.

[8] R. Barate et al., Eur. Phys. J. C 2, 395 (1998).

[9] H. Albrecht et al. (ARGUS Collaboration), Phys. Lett. B 292, 221 (1992).

[10] G. Abbiendi et al. (OPAL Collaboration), Phys. Lett. B 492 , 23 (2000).

[11] W. Bacino et al. (DELCO Collaboration), Phys. Rev. Lett. 41, 13 (1978).

[12] E. V. Abakumova et al., Nucl. Instrum. Methods Phys. Res., Sect. A 659, 21 (2011).

[13] M. N. Achasov et al., Chin. Phys. C 36, 573 (2012).

[14] M. Ablikim et al., Nucl. Instrum. Methods Phys. Res., Sect. A 614, 345 (2010).

[15] P. Rullhusen, X. Artru, and P. Dhez, Novel Radiation Sources Using Relativistic Electrons (World Scientific, Singapore, 1998).
[16] L. D. Landau and E. M. Lifshitz, Relativistic Quantum Mechanics (Pergamon, New York, 1971).

[17] Giovanni Balossini, C. M. Carloni Calame, G. Montagna, O. Nicrosini, and F. Piccinini, Nucl. Phys. B758, 227 (2006).

[18] S. Agostinelli et al. (GEANT4 Collaboration), Nucl. Instrum. Methods Phys. Res., Sect. A 506, 250 (2003).

[19] Z. Y. Deng et al., HEP\&NP 30, 371 (2006).

[20] S. Jadach, B. F. L. Ward, and Z. Was, Comput. Phys. Commun. 130, 260 (2000); Phys. Rev. D 63, 113009 (2001).

[21] D. J. Lange et al., Nucl. Instrum. Methods Phys. Res., Sect. A 462, 152 (2001).

[22] J. C. Chen, G. Huang, X. Qi, D. Zhang, and Y. Zhu, Phys. Rev. D 62, 034003 (2000).

[23] K. Yu. Todyshev, arXiv:0902.4100.

[24] F. A. Berends, K. J. F. Gaemers, and R. Gastmans, Nucl. Phys. B57, 381 (1973); F. A. Berends and G. J. Komen, Phys. Lett. 63B, 432 (1976).

[25] E. A. Kuraev et al., Sov. J. Nucl. Phys. 41, 466 (1985); O. Nicrosini and L. Trentadue, Phys. Lett. B 196, 551 (1987); F. A. Berends, G. Burgers, and W. L. Neerven, Nucl. Phys. B297, 429 (1988); B304, 921 (1988).

[26] P. Ruiz-Femenía and A. Pich, Phys. Rev. D 64, 053001 (2001).

[27] G. Rodrigo, A. Pich, and A. Santamaria, Phys. Lett. B 424, 367 (1998).

[28] M. B. Voloshin, Phys. Lett. B 556, 153 (2003).

[29] X. H. Mo, Nucl. Phys. B, Proc. Suppl. 169, 132 (2007); Y. K. Wang et al., HEP\&NP 31, 325 (2007).

[30] A. Pich, Acta Phys. Pol. B 38, 3449 (2007). 